la revue La revue pour l'histoire du CNRS

POUR LHISTORE DU CNRS $10 \mid 2004$

Penser la pensée. Les sciences cognitives

\title{
La création de l'Institut des sciences cognitives du CNRS (1992-1998)
}

Marc Jeannerod

\section{(2) OpenEdition}

\section{Journals}

Édition électronique

URL : https://journals.openedition.org/histoire-cnrs/585

DOI : $10.4000 /$ histoire-cnrs. 585

ISSN : 1955-2408

Éditeur

CNRS Éditions

Édition imprimée

Date de publication : 5 mai 2004

ISBN : 978-2-271-06145-4

ISSN : $1298-9800$

\section{Référence électronique}

Marc Jeannerod, «La création de l'Institut des sciences cognitives du CNRS (1992-1998) », La revue pour l'histoire du CNRS [En ligne], 10 | 2004, mis en ligne le 18 janvier 2007, consulté le 20 mai 2021. URL : http://journals.openedition.org/histoire-cnrs/585; DOI : https://doi.org/10.4000/histoire-cnrs. 585

Ce document a été généré automatiquement le 20 mai 2021.

Comité pour l'histoire du CNRS 


\title{
La création de l'Institut des sciences cognitives du CNRS (1992-1998)
}

\author{
Marc Jeannerod
}

1 La création d'un Institut des sciences cognitives par le CNRS au cours des années 1990 représente l'aboutissement d'un long processus marqué par un vaste débat. D'une part, le contenu même de ce domaine de recherche posait le problème épistémologique d'une approche scientifique du fonctionnement de l'esprit et des moyens à mettre en œuvre pour y parvenir. D'autre part, l'introduction d'un nouveau domaine scientifique posait le problème institutionnel de sa délimitation et de ses relations avec les autres disciplines concernées. Ce débat a largement influencé les choix scientifiques et la structure même de la nouvelle formation au moment de sa création et au début de son fonctionnement. Ce même débat, toutefois, ne peut être détaché du contexte scientifique et institutionnel de la décennie précédente au cours de laquelle cette nouvelle voie de recherche a lentement pénétré le milieu scientifique français. À ce titre, la structuration des sciences cognitives en France et la création de l'Institut, qui n'en est que l'un des aspects, posent un problème plus vaste, celui de la réponse de l'institution, et singulièrement du CNRS, à une innovation scientifique.

L'émergence des sciences cognitives en France

2 La décision de création de l'Institut remonte, comme on le verra plus loin, au début des années 1990. Cette décision faisait suite à un ensemble d'initiatives, prises par les instances responsables de la recherche dans la seconde moitié des années 1980, qui avaient clairement insisté sur la nécessité d'une orientation interdisciplinaire des recherches dans divers domaines, dont celui de l'étude de la cognition. Parmi ces initiatives, l'action de recherche intégrée (Ari) sur les « sciences de la communication ", lancée par le département des sciences humaines et sociales (SHS) du CNRS et pilotée par Dominique Wolton, a joué un rôle de précurseur dès 1984. Une action thématique programmée (ATP), intitulée « intelligence artificielle et sciences cognitives », avait été créée dans le cadre de cette initiative et avait financé quelques projets. L'action de Dominique Wolton favorisait la tendance orthodoxe des sciences cognitives en donnant la primauté à l'interface entre l'intelligence artificielle et les sciences du langage et de 
la communication, tout en excluant les neurosciences. Un autre groupe de travail avait toutefois été mis en place au même moment par le CNRS sur «informatique et neurosciences ». Sous la responsabilité de Claude Kordon, ce groupe devait déboucher à l'automne 1985 sur une proposition, non suivie d'effet à l'époque, de "réseau sans murs» regroupant un petit nombre d'équipes de neurobiologie, d'intelligence artificielle et d'épistémologie.

3 Au niveau européen, le programme Fast (Forecasting and Assessment in Science and Technology), un programme expérimental de prévision et d'évaluation dans le domaine de la science et de la technologie, avait fixé parmi ses objectifs pour les années 1985-1986 une réflexion sur les perspectives des sciences cognitives. Dans le document de présentation, les sciences cognitives étaient définies comme une approche ayant pour objet l'étude de " l'intelligence humaine, y compris sa structure mathématique, sa réalisation psychologique, son substrat neuronal ». Les disciplines concernées, d'après ce document, relevaient de la neuropsychologie, de la psychologie expérimentale, de la linguistique théorique, de la philosophie analytique, de la cybernétique. L'impact potentiel des sciences cognitives dans le domaine des relations homme-machine était par ailleurs mis en avant. Un groupe de réflexion présidé par Michel Imbert organisa une rencontre européenne (European Cognitive Science, Paris, 9 et 10 mai 1985) rassemblant une soixantaine de participants et coordonna la rédaction d'un rapport où l'on voit clairement apparaître une distinction "hétérodoxe » entre intelligence biologique (dont l'étude doit être favorisée) et intelligence artificielle (qui appartient au monde de la technologie) ${ }^{1}$. Cette distinction sera reprise plus loin.

4 C'est finalement au niveau ministériel que sera prise l'initiative décisive : dans le cadre d'une action "sciences de la cognition", un rapport est demandé à Jean-Pierre Changeux qui réunit un comité scientifique et formule des recommandations sur la façon de structurer le domaine de recherche représenté par les sciences cognitives. Ce rapport insistait particulièrement sur le rôle de la psychologie cognitive et des neurosciences comme bases permettant de structurer un ensemble plus vaste de disciplines: la psychologie sociale, l'économie politique, la linguistique générative, l'anthropologie cognitive et la philosophie, d'une part, l'étude des réseaux de neurones artificiels, d'autre part. Le chapitre sur les "actions à entreprendre " contenait un ambitieux programme qui sera, pour l'essentiel, réalisé : ce programme incluait la création d'une action concertée à long terme sur les sciences cognitives, la mise en place de bourses de formation, la création d'enseignements spécifiques, d'une bibliothèque. Le rapport préconisait enfin la création d'instituts de sciences cognitives "où l'interdisciplinarité caractéristique des sciences cognitives puisse relever de la pratique quotidienne » et où les compétences et les moyens techniques puissent être mis en commun. Il apparaissait nécessaire aux rédacteurs du rapport que ce type d'institut soit créé à partir de bâtiments neufs et de personnels nouveaux. "Les expériences qui ont réussi dans le passé sont en général celles qui ont fait rupture avec les structures existantes. » Les recommandations formulées dans ce rapport seront déterminantes pour la naissance de l'Institut de Lyon'2.

5 Cet ensemble d'initiatives prises dans les années 1980 en faveur d'une implantation et d'un développement des sciences cognitives en France faisait écho au courant scientifique, presque exclusivement nord-américain, qui a vu l'émergence de ce domaine une trentaine d'années auparavant. Les premiers faits marquants qui constituent ce qu'on appelle maintenant la «révolution cognitive» se sont en effet 
produits entre 1948 et 1950: il s'agissait de rencontres (le Hixon Symposium, les conférences de la Macy Foundation) entre personnalités scientifiques appartenant à différents domaines scientifiques dont les mathématiques, la linguistique, la psychologie ou la neurophysiologie ${ }^{3}$. Les thèmes de discussion abordés au cours de ces rencontres seront évoqués plus loin dans cet article, lorsqu'il sera question de la définition du programme scientifique du nouvel institut. Au cours des années 1960, toujours aux États-Unis, les premiers pas vers l'institutionnalisation des recherches sur la cognition avaient été marqués par la création d'un Center for Cognitive Studies à l'université Harvard, ainsi que par la parution d'ouvrages fondateurs, comme Cognitive Psychology d'Ulrich Neisser ${ }^{4}$ ou les premiers travaux d'Herbert Simon ${ }^{5}$. C'est finalement en 1976 que le programme de l'Alfred P. Sloan Foundation vit le jour, destiné à favoriser, par des financements, des recherches à l'intersection entre plusieurs approches de la cognition 6 .

6 On voit bien, si l'on prend cette date de 1976 comme référence pour le début institutionnel des sciences cognitives aux États-Unis, que les initiatives françaises dans le même domaine souffraient d'un retard de l'ordre de dix ans, sinon pour ce qui est de la prise de conscience du problème, du moins pour ce qui est du passage aux réalisations pratiques. Un programme interdisciplinaire de recherche intitulé " cognisciences ${ }^{7}$ ", dirigé par André Holley, est en effet lancé par le CNRS en 1990 : c'est dans ce cadre que sont financés les premiers réseaux régionaux de sciences cognitives, qui débutent leur activité la même année. Quant à l'Institut des sciences cognitives, sa création fut décidée au cours de l'année $1992^{8}$.

7 Le caractère tardif de ces réalisations ne tenait pourtant pas à un déficit d'information des milieux scientifiques concernés. Dès les années 1970, en effet, des précurseurs avaient attiré l'attention de la communauté par des actions marquantes mettant en contact les chercheurs français avec la réalité internationale. C'est ainsi qu'en juin 1975 fut organisée par le Centre Royaumont pour une science de l'homme, à l'abbaye du même nom, une rencontre entre deux des figures indiscutées de l'étude de la cognition, Jean Piaget et Noam Chomsky. Autour des deux protagonistes, biologistes, mathématiciens, psychologues et philosophes avaient pu confronter leurs idées dans une ambiance qui n'était peut-être pas très différente de celle des rencontres de la Macy Foundation ${ }^{9}$. Cinq ans plus tard, en juin 1980, l'abbaye de Royaumont était le théâtre d'une nouvelle manifestation financée, cette fois, par le CNRS, sous la forme d'un colloque international rassemblant les principaux acteurs de la psychologie cognitive. La lettre d'invitation rédigée par Jacques Mehler, l'organisateur du colloque, précisait que son but était « d'explorer les principales approches du traitement cognitif [...] et de définir les domaines dans lesquels les progrès les plus importants peuvent être attendus dans les cinq ou dix ans à venir ${ }^{10} »$. Jacques Mehler était par ailleurs, on le sait, le fondateur de la revue Cognition, une des principales revues de psychologie cognitive, dont les premiers numéros furent publiés en 1972.

8 La prudence du CNRS et sa lenteur à réagir dans ce domaine tiennent à plusieurs facteurs. Le moindre n'est pas la division du CNRS et des autres instances scientifiques françaises représentatives (Comité national, $\mathrm{CNU}$ ) en sections scientifiques spécialisées. Comment, en effet, prendre en compte un champ de recherche qui émargerait simultanément, pour ce qui est du CNRS, aux départements des sciences de la vie, des sciences de l'homme et de la société et des sciences pour l'ingénieur? Où situer dans la classification des sciences, par qui faire évaluer et comment financer un projet qui se 
serait réclamé de ce domaine? La nécessaire interdisciplinarité, même prônée par maints comités de réflexion et maints rapports, n’a été reconnue, sous la forme de sections "transversales» ou d'un nouveau département du CNRS, qu'au début des années 2000 ! Au cours de la décennie précédente, il n'a été question que de l'impérialisme supposé d'une discipline vis-à-vis des autres et de la revanche à prendre sur cette discipline lors de la prochaine alternance. C'est ainsi qu'au programme " cognisciences", accusé de favoriser les neurosciences, a succédé le programme « sciences de la cognition » dominé par l'intelligence artificielle, lequel a laissé place au programme "Cognitique » dominé par les sciences humaines. Un autre facteur, plus indirect, de cette lenteur à répondre à l'émergence d'une nouvelle discipline est l'absence en France de fondations privées suffisamment structurées et, surtout, suffisamment puissantes financièrement. De telles fondations, on l'a vu, ont joué ailleurs un rôle déterminant d'anticipation et de stimulation des instances institutionnelles.

La discussion des choix scientifiques du nouvel institut

Il est classiquement admis par les tenants du modèle cognitiviste orthodoxe que les processus cognitifs doivent être considérés indépendamment de toute incarnation particulière. On retrouve ce dogme de l'irréductibilité de la cognition à son support dans de nombreuses formulations disciplinaires: en informatique, les opérations logiques du software ne doivent rien à la constitution du hardware; en neurosciences, les états mentaux devaient pouvoir être décrits séparément de la constitution du cerveau. Un même état cognitif (une même opération logique) peut être l'objet de plusieurs réalisations différentes selon le système dans lequel il est implémenté (le concept de multiple realizability), mais qui aboutissent toutes au même résultat. Il serait donc illusoire, en suivant cette thèse, de chercher à faire une distinction, pour reprendre les termes du rapport de Michel Imbert, entre les processus cognitifs réalisés par des machines (une cognition artificielle) et ceux réalisés par des systèmes biologiques, comme des cerveaux (la cognition naturelle).

L'étendue du domaine des sciences cognitives excluait cependant d'emblée la possibilité d'une couverture exhaustive au sein du nouvel institut. Le choix a donc été fait de privilégier la cognition naturelle par rapport à la cognition artificielle. Les arguments avancés pour justifier ce choix étaient de plusieurs ordres. D'un côté, il paraissait illusoire de vouloir créer un nouveau pôle attractif dans le domaine de l'intelligence artificielle en dehors des centres existants et possédant déjà une véritable masse critique; d'un autre côté, l'étude des processus cognitifs en relation avec le support biologique paraissait correspondre à une demande dans plusieurs domaines critiques dont l'état d'avancement justifiait une approche concertée par des spécialistes de disciplines différentes mais travaillant en synergie et sous le même toit. Les mécanismes du langage, ceux de la représentation de l'intention et de l'action, les mécanismes de la reconnaissance et de la mémorisation, les émotions, figurent parmi ces problèmes.

11 On notera que tous ont un impact direct sur des thèmes de société, qu'il s'agisse du développement cognitif et de l'éducation, des dysfonctionnements de la cognition et de la pathologie mentale, de la représentation des connaissances et de la communication. Ainsi, à côté de l'impact déjà clairement reconnu des sciences cognitives sur la technologie et l'industrie, se dessinaient de nouveaux domaines où elles semblaient appelées à jouer un rôle important, ceux de la santé et de l'éducation. 
12 À l'époque des discussions sur le programme scientifique (1992-1993), toutefois, ces arguments en faveur d'un programme limité à l'étude de la cognition naturelle étaient apparus relativement secondaires par rapport à la violation supposée de la notion d'une permutabilité absolue entre tous les domaines et toutes les expressions de la cognition. La communauté représentée par le département des sciences pour l'ingénieur (SPI) du CNRS s'était montrée, comme on pouvait s'y attendre, particulièrement ferme sur ce point. Le fait que le choix portant sur la cognition naturelle ait été par la suite confirmé devait d'ailleurs aboutir à un désengagement complet du département SPI vis-à-vis du financement de l'Institut.

13 À l'opposé, le principe de permutabilité des différentes formes de cognition se heurtait à d'autres critiques, tout aussi virulentes, de la part des spécialistes des domaines concernés qui craignaient qu'une interdisciplinarité étendue à un trop grand nombre de secteurs scientifiques n'aboutisse à un programme confus et inefficace. Il s'agissait là, soit d'un doute persistant sur l'existence même des sciences cognitives, considérées comme un assemblage artificiel sans but défini et destiné avant tout à créer de nouvelles opportunités tactiques en matière de postes ou de crédits de recherche; soit d'une ignorance des objectifs et du programme scientifique de ce domaine émergent et des questions épistémologiques qu'il soulevait. Les critiques émises quant à l'idée de création d'un Institut des sciences cognitives portaient donc également sur des aspects plus théoriques. Les uns redoutaient une dérive des sciences cognitives vers une réduction physicaliste, qui aurait risqué de masquer ou de déformer la réalité des processus cognitifs en les ramenant à des équations mathématiques. D'autres, au contraire, ceux qui pensent que le but de toute science naturelle est d'expliquer les phénomènes au niveau le plus élémentaire possible, jugeaient l'approche cognitive trop globale et détachée de la base cellulaire et moléculaire du fonctionnement nerveux. D'autres, enfin, considéraient que l'étude même des états mentaux et des représentations était entachée de trop de subjectivisme pour pouvoir prétendre à une description réellement scientifique de ces problèmes.

Une grande partie de ces critiques, particulièrement celles qui portaient sur la notion d'interdisciplinarité ou celles qui témoignaient de la crainte d'une évolution vers le physicalisme, tiraient leur justification de la conception orthodoxe du domaine des sciences cognitives tel qu'il s'était structuré au moment de sa formation aux États-Unis dans l'immédiat après-guerre. Quarante ans plus tard, ces arguments perdaient de leur force $\mathrm{du}$ fait des profondes transformations qu'avaient subies les disciplines constitutives. Les promoteurs les plus influents des sciences cognitives à leur début, mathématiciens pour la plupart, avaient axé leurs efforts sur une définition des processus cognitifs et du traitement de l'information en termes d'opérations sur des symboles. Le système nerveux lui-même était conçu comme un système de traitement fonctionnant sur ce principe de «tout ou rien». Il paraissait donc logique à ces précurseurs d'assimiler le cerveau à d'autres systèmes, existants ou à venir, opérant sur le même type de traitement symbolique : penser ou raisonner ne serait rien d'autre que calculer, pour reprendre l'adage classique. Par la suite, la déception engendrée par l'évolution de l'intelligence artificielle, d'une part, et le développement explosif des neurosciences, d'autre part, ont radicalement transformé les idées issues de la révolution des années 1950 . Dès les premières années, d'ailleurs, des doutes s'étaient élevés sur l'analogie du système cognitif et des programmes informatiques, trop peu enclins, selon Ulrich Neisser, à la distraction et à l'émotion. De même, Karl Lashley 
posait dès les années 1950 la question de savoir si la science cognitive devait utiliser l'ordinateur pour comprendre le savoir humain, ou le cerveau pour comprendre l'ordinateur! En fait, comme le fait remarquer Howard Gardner dans son Histoire de la révolution cognitive, publiée en 1985, l'application rigoureuse des modèles tirés de l'informatique a paradoxalement aidé les scientifiques à comprendre en quoi les processus cognitifs des êtres humains ne ressemblent pas à ceux des ordinateurs. "Le type de vision systématique, logique et rationnelle de la cognition humaine qui a envahi la littérature des débuts de la science cognitive ne décrit pas convenablement la pensée et le comportement humains ${ }^{11}$.»

Le fait est que la comparaison entre cerveau et ordinateur révèle plus de différences que de similitudes. En effet, les ordinateurs fonctionnent sur le principe du tout ou rien, en alignant très rapidement des symboles digitaux produits par des circuits intégrés. Dans le cerveau, en revanche, l'information est le produit de l'intégration d'une multitude de variables physico-chimiques - des courants ioniques traversant les canaux de la membrane des neurones. L'activité d'un neurone qui résulte de ces courants ioniques est bien, dans une certaine mesure, du type tout ou rien, mais le neurone n'est pas isolé, il fonctionne au sein de populations où de nombreux éléments agissent en parallèle. Le résultat final de ce traitement est donc de nature probabiliste, si bien que l'effet d'un seul neurone est négligeable et que, contrairement à ce qui se passe dans un ordinateur, aucun neurone n'est nécessaire au bon fonctionnement de l'ensemble.

16 Des modèles physiques plus réalistes du traitement neuronal ont été imaginés plus récemment: ce sont les modèles connexionnistes qui possèdent certaines des caractéristiques du réseau nerveux biologique, en particulier celle de traiter l'information selon des voies parallèles (on parle de réseaux "neuromimétiques »). Leur existence même, toutefois, et le fait qu'ils sont éventuellement capables de donner des réponses à des questions cognitives, sont en contradiction avec la notion de traitement symbolique, fondé sur un traitement séquentiel, qui représente l'attribut principal du traitement de l'information dans la version cognitiviste classique. Le fonctionnement même de ces réseaux s'inspire d'ailleurs des connaissances acquises au cours des dernières décennies sur le fonctionnement du système nerveux : traitement de type analogique plutôt que digital, importance de l'organisation spatiale, introduction de la notion de "poids synaptique " pour rendre compte d'un filtrage partiel des informations au niveau des connexions, autant de caractéristiques qui distinguent définitivement ces réseaux du mode de fonctionnement des ordinateurs classiques.

Le choix de la cognition naturelle avait donc pour corollaire une forte participation des neurosciences. L'évolution d'une partie des neurosciences (les neurosciences " cognitives») a créé des conditions nouvelles pour l'étude des processus mentaux. Sous l'influence de la psychologie, le vocabulaire cognitif a pu être réintroduit dans les paradigmes expérimentaux pour l'étude des fonctions du cortex cérébral. Grâce à l'introduction des techniques de neuro-imagerie, le niveau molaire de description a pu se substituer au niveau moléculaire dominant. Des concepts tels que ceux d'ensembles de neurones ou de réseaux sont apparus et ont acquis droit de cité. Ils permettent d'envisager l'élaboration d'une théorie dynamique du fonctionnement nerveux compatible avec le niveau des phénomènes cognitifs. 
18 Un autre choix déterminant aura été d'introduire la philosophie comme élément constitutif du programme initial ${ }^{12}$. L'introduction dans l'étude de la cognition naturelle d'une dimension philosophique a permis de rendre opérationnel le concept abstrait de «naturalisation » des états mentaux. La fonction principale du système cognitif est de fabriquer des représentations. Chacun peut se représenter une personne, un objet, une action à entreprendre, une situation, etc., en utilisant les données fournies par les organes des sens ou les données stockées dans le système cognitif et qui constituent nos connaissances et nos croyances. La notion de naturalisation postule que ces représentations sont des objets naturels, et qu'à ce titre, elles possèdent, comme n'importe quel autre objet, deux types de propriétés: d'une part, des propriétés physiques et d'autre part, des propriétés sémantiques. Une intention, par exemple, possède à la fois des propriétés physiques (l'activation d'un ensemble de circuits nerveux dans le cerveau) et des propriétés sémantiques, le contenu de cette intention (le but à atteindre). De même, on peut créer à l'intérieur d'un ordinateur la représentation (d'une action à exécuter, par exemple) et donner à la machine des instructions pour l'exécution de cette action (un programme). Au moment d'être exécuté, ce programme existe dans la machine comme un certain état du réseau électronique (propriété physique) en même temps qu'il possède un contenu sémantique pour mener à bien l'action désirée.

19 Les propriétés sémantiques sont largement indépendantes du support physique : si l'on prend comme exemple le texte de cet article, ses propriétés sémantiques seront les mêmes, que le texte soit imprimé sur un support papier ou inscrit sur un disque dur. Toutefois, ces propriétés ne peuvent exister indépendamment d'un support. En d'autres termes, il n'y a pas de sens qui flotte entre deux incarnations. Le problème que se posent les chercheurs en sciences cognitives est donc de savoir comment des propriétés non sémantiques (physiques) peuvent produire des propriétés sémantiques, ou, à l'inverse, comment le contenu sémantique d'une représentation peut être «naturalisé » en termes de propriétés physiques du réseau nerveux. Cette naturalisation vise à faire de l'activité mentale, celle qui constitue nos représentations, la production, ou la conséquence, de l'activité cérébrale : ce n'est qu'à partir de là qu'on peut tenter de déterminer les effets de ces représentations sur le comportement; ce n'est que si les états mentaux sont des états cérébraux qu'on peut envisager leurs effets sur les autres parties du cerveau, les muscles ou les organes végétatifs ${ }^{13}$.

Le programme initial du nouvel Institut a réuni des équipes autour de cinq chapitres principaux: les sciences du langage, la reconnaissance des formes et la mémoire, la planification de l'action, l'organisation spatiale du comportement, et enfin la modélisation des processus cognitifs. Un tel programme se veut évidemment évolutif et modifiable.

21 Une des conséquences du programme de naturalisation des états mentaux, qui constitue le cœur de l'étude de la cognition naturelle, est qu'il introduit des contraintes biologiques dans le fonctionnement cognitif. Nos représentations doivent, d'une manière ou d'une autre, être commensurables à notre cerveau. L'esprit (humain) possède une structure qui reflète ces contraintes biologiques et nos représentations dépendent de cette structure pour leur forme et leur expression. Peut-on, en poursuivant cette analyse, avancer que le contenu sémantique de nos états mentaux devrait être conditionné par les mécanismes nerveux qui les produisent? Que ce contenu devrait obéir au processus de sélection à l'œuvre dans l'évolution collective et 
individuelle de l'espèce humaine ? Il s'agit là de pures conjectures. C'est pourtant bien la sélection naturelle qui a permis l'apparition des capacités cognitives complexes que sont l'élaboration de représentations ou l'utilisation du langage. Au cours du développement individuel, les processus de maturation cognitive fonctionnent aussi sur le mode de la sélection : en se spécialisant pour la phonologie d'une langue, l'enfant diminue sa capacité d'en apprendre une autre. Un «darwinisme mental » viendrait compléter le travail de l'évolution. Une des orientations actuelles qui découle de cette notion consiste à rechercher l'existence, chez les espèces qui ont précédé l'homme, de précurseurs des états cognitifs humains.

Ainsi se dessine une nouvelle définition des sciences cognitives, centrée, selon les termes de Michel Imbert, sur l'étude de l'intelligence humaine, de sa structure formelle à son substratum biologique. Cette définition repose sur la conviction que "seule une association étroite entre sciences du cerveau, psychologie, linguistique, informatique, anthropologie et philosophie [...] peut apporter des réponses nouvelles, c'est-à-dire issues de recherches empiriques, aux questions traditionnelles concernant la nature de l'esprit humain ${ }^{14} »$. L'avenir dira si cette proposition peut devenir effective et opérationnelle dans le cadre quotidien d'un laboratoire où les chercheurs, au-delà des considérations théoriques, peuvent interagir sur des objectifs scientifiques limités et bien définis.

L'auteur remercie André Holley pour les indispensables précisions qu'il lui a fournies sur les débuts des sciences cognitives en France.

\section{NOTES}

1.Voir M. Imbert, P. Bertelson, R. Kempson, D. Osherson, H. Schnelle, N.A. Streitz, A. Thomassen et P. Viviani (éd.), Cognitive Science in Europe, Berlin, Springer Verlag, 1987. 2.Rapport sur les sciences cognitives rédigé par un comité scientifique présidé par Jean-Pierre Changeux, remis aux ministres de l'éducation et de la Recherche en 1988. D'autres rapports plus tardifs furent également rédigés sur les sciences cognitives, notamment par Alain Berthoz (1991), Bernard Guibert (1992) et Jean-Gabriel Ganascia (1995). Voir à ce sujet B. Chamak, «Étude de la construction d'un nouveau domaine : les sciences cognitives. Le cas français ", thèse de doctorat, université Paris VII, 1997. 3.J.-P. Dupuy, Aux origines des sciences cognitives, Paris, La Découverte, 1994. 4.U. Neisser, Cognitive psychology, New York, Appleton-Century-Croft, 1967.

5.H. A. Simon, The Science of the Artificial, Cambridge, MIT Press, 1969 (trad. fr. : La Science des systèmes, science de l'artificiel, Paris, L'Épi, 1974) et Models of my Life, New York, Basic Books, 1992.

6.Un rapport, publié en 1978 par Keyser, Miller et Walker, préconisait de favoriser les recherches impliquant plusieurs des six disciplines de base des sciences cognitives : philosophie, linguistique, psychologie, neurosciences, anthropologie et sciences du calcul. Les interactions entre ces disciplines existant en 1978 étaient représentées sous 
la forme d'une célèbre figure hexagonale.Voir G. A. Miller, « The Cognitive Revolution: a Historical Perspective », Trends in Cognitive Science, 7, 2003, p. 141-144.

7.Les principales directions du programme « cognisciences » sont présentées dans un numéro spécial du Courrier du CNRS ( ${ }^{\circ} 79$, octobre 1992). Il y est affirmé que les « études théoriques et recherches expérimentales joignent leurs efforts pour comprendre le fonctionnement du cerveau et de l'esprit... ».

8.La lettre de mission adressée au responsable de projet par François Kourilsky, alors directeur général du CNRS, est datée du 12 juin 1992.

9.M. Piatelli-Palmarini, (éd.), Théories du langage, théories de l'apprentissage. Le débat entre Jean Piaget et Noam Chomsky, Paris, Seuil, 1979.

10.J. Mehler, E. C. T. Walker et M. Garrett (éd.), Perspectives on Mental Representation. Experimental and Theoretical Studies of Cognitive Processes and Capacities, Hillsdale, LEA, 1982.

11.H. Gardner, The Mind's New Science. A History of the Cognitive Revolution, New York, Basic Books, 1985. Trad. fr. : Histoire de la révolution cognitive. La nouvelle science de l'esprit, Paris, Payot, 1993, p. 60.

12.Ce choix a d'ailleurs été matérialisé par la création, en 1995, et donc avant l'ouverture de l'Institut proprement dit, d'une équipe postulante (EP 100) intitulée «Modularité des processus cognitifs », dirigée par le philosophe Pierre Jacob. L'EP 100, implantée dans des locaux prêtés par l'université Claude Bernard, a permis aux premiers chercheurs du futur Institut de se regrouper autour d'un séminaire interdisciplinaire associant systématiquement un spécialiste d'une des principales disciplines constitutives des sciences cognitives à un philosophe.

13.Voir D. Andler, "Le cognitivisme orthodoxe en question ", Cahiers du CREA, $\mathrm{n}^{\circ} 9$ : Cognition et complexité, 1986, p. 9-106 ; P. Jacob, Pourquoi les choses ont-elles un sens?, Paris, Odile Jacob, 1997.

14.M. Imbert, « Neurosciences et sciences cognitives », in D. Andler (éd.), Introduction aux sciences cognitives, Paris, Gallimard, 1992, p. 49.

\section{RÉSUMÉS}

The Creation of the CNRS' Cognitive Sciences Institute

In June 1992 the CNRS decided to create an Institute devoted to researches in cognitive sciences, located in Lyon. Officially created in January 1997, the new building was opened in April 1998. This creation represented the logical outcome of the national research program in cognitive sciences which supported interdisciplinary teams, located outside Paris. The urgency to fill the gap which had its origins in the rapid development of these sciences in the United States constituted the main objective. To attain this aim, it was necessary to create strong interactions between disciplines concerned by artificial intelligence and those, then scattered, that tried to describe human mind structure and functions: neurosciences, psychology, psychopathology, language sciences, philosophy of mind. The main strategy of this new Institute was to bring together researchers specialised in different disciplines in order that they worked on the same problems like action planning, form recognition or space representation. The efforts deployed to 
reach this objective showed the structural constraints without entirely overcome them. These constraints are originated in the CNRS' structured in several departments and in the divisions caused by the different French organisms being in charge of research.

\section{AUTEUR}

\section{MARC JEANNEROD}

Marc Jeannerod est professeur à l'université Claude Bernard de Lyon et membre de l'Académie des sciences. Son dernier ouvrage paru est La Nature de l'esprit, Paris, Odile Jacob, 2002. 